\title{
Threat Assessment of Aerial Target Based on Modified PSO Optimized BP Neural Network
}

\author{
$\mathrm{Xia} \mathrm{Chen}^{\mathrm{a}, \mathrm{b}}$ and Tuo Wang ${ }^{\mathrm{a}}$ \\ ${ }^{a}$ School of Economics and Management, Shenyang Aerospace University 1101361, \\ ShenYang, China; \\ ${ }^{b .}$ School of Automation, Shenyang Aerospace University 1101361, Shen Yang, \\ China; \\ ${ }^{a}$ School of Economics and Management, Shenyang Aerospace University \\ 1101361, ShenYang, China; \\ xiachen1108@163.com,wangtuo316@163.com
}

\begin{abstract}
Aerial target threat assessment is to infer degree of target threat to us through comprehensive analysis of the target information, then sort on target, to provide the basis for commander of task allocation and tactical decision; it is the core of the air defense combat command and control system. Consider the aerial target threat assessment problem, A target threat assessment method is proposed by using Modified Particle Swarm Optimization (MPSO) algorithm to optimize the BP neural network. Firstly, this paper carries out analysis on the main factors affecting target threat assessment, then introduces the basic principle of BP neural network and particle swarm optimization (PSO) algorithm and its improvement, and the MPSO-BP neural network assessment model is constructed. Finally, the model is verified by simulation. Results show that the error of this method is obviously less than that of BP neural network and PSO-BP neural network assessment method and has a good evaluation effect.
\end{abstract}

Keywords: aerial target; threat assessment; BP neural networks; Particle Swam Optimization algorithm

\section{Introduction}

The rhythm of modern air combat is growing faster with the development of information and intelligence, this puts forward higher requirements for real-time and accuracy of target threat assessment. Therefore, how to evaluate the threat of air target timely and accurately is a research topic which has great theoretical value and realistic meaning. At present, the study of air target assessment has obtained some results [1-5]. Cheng [1] considering that the information of target attribute is sometimes uncertain and based on analysis to methods for selecting target attributes and determining their weights, put forward a decision-making method combining precise real-number type index with fuzzy interval type index. An advanced Analytic Hierarchy Process (AHP) is which is used for multi-target threaten degree sequencing is gotten by structure of decision matrix and the variation of weight through analytic hierarchy process in [2], etc. In recent years, the intelligent technology represented by neural network is widely used in image recognition, fault diagnosis, forecast and other fields. Therefore, the scholars turned to the research field of neural network which has obvious advantages in dealing with non-linear problems. In order to solve the problem of beyond visual range formation cooperative attack, Xiao [3] established a multi-objective distributed neural network decision model, according to the situation assessment matrix, the effective attack order is obtained by Hopfield neural network. Nian [4] takes the air defense operation actual needs as a 
starting point, from the view point of the whole information, on the base of improving the traditional BP algorithm, aerial threat evaluation model for ship formation is built. Chen [5] established a target thread model based on the factors of space situation and air combat capacity of target, such as altitude, distance, speed and angle, and proposed a target threat level assessment method based on PSO and BP algorithm, to estimate the threat level of aerial target, etc. PSO algorithm is a kind of swarm intelligence optimization algorithm, which is simple and easy to implement, but when applied to high-dimensional complex problems often appear the phenomenon of premature convergence and slow late convergence speed, which can not guarantee convergence to the optimal value. In order to overcome the above shortcomings of PSO algorithm, MPSO algorithm which introducing mutation operation and linear decreasing inertia weight is proposed, and a target threat assessment model is established by using MPSO algorithm to optimize the BP neural network. So far, research of target threat assessment based on MPSO-BP neural network has not been reported in the literature.

In response to these phenomena, this paper analyzed the main factors affecting target threat assessment firstly, and then introduces the related theory of BP neural network and PSO algorithm and its improved theory; target threat assessment model is constructed by using MPSO-BP neural network. Finally, the feasibility and validity of this method are proved with example analysis by using MATLAB tool.

\section{Influencing Factors Analysis of Target Threat Assessment}

Combined with the actual situation of air defense combat, when making air target threat evaluation, this paper mainly considers the following factors:

\subsection{Target Velocity $(V)$}

The velocity of the target is the vector synthesis of its approximation rate and its lateral velocity, directly affect the target threat assessment. Even if the same type of target, if the flight speed is different, their threat degree is also not the same. In general, the faster the flight speed, the greater the threat degree.

\subsection{Target Type $(K)$}

Different types of target have different flight speed and attack ability, the threat level to protected target is also different. In this paper, the target type is divided into three kinds of reconnaissance plane, small target (such as tactical ballistic missiles, stealth aircraft) and large target (such as bomber, fighter bomber). In general, the threat degree of small target is the biggest, and the threat degree of reconnaissance plane is the least. In order to facilitate quantitative study, in this paper, the target threat attribute is quantified by G. A. Miller's nine levels quantization theory [6], the reconnaissance plane, small target and large target were quantified for 3,5 and 8 .

\subsection{Heading Angle of Target $(\theta)$}

Heading angle of target is the angle between the target advancing direction and the direction of target's position to the protected target's position. In general, the smaller the heading angle, the more likely the target suddenly appeared, and the threat is also greater.

\subsection{Target Height $(H)$}

When the target is far, the threat of flying height to our side is not obvious, but the target that suddenly appeared in low altitude will be a great threat to us. In this paper, the index is quantified by G. A. Miller's nine levels quantization theory, the 
ultralow altitude, low altitude, medium altitude and high altitude were quantified for 2, 4, 6 and 8 .

\subsection{Target Distance $(D)$}

The closer the distance between the incoming target and the protected target, the shorter the defense time, the greater the incoming target threat level. Contrariwise, the farther the distance between the incoming target and the protected target, the attack intent of incoming target is not more obvious, the lower the threat level.

\subsection{Interference Capability of Target $(C)$}

Target interference capability is one of the typical counter measures of enemy air raid formation, it can be divided into four kinds: very weak, weak, medium and strong. Generally, the target interference capability is stronger, the threat degree is greater. In the paper, this index is quantified by G. A. Miller's nine levels quantization theory, the very weak, weak, medium, strong was quantified for 2, 4, 6 and 8.

\section{BP Neural Network}

Back-Propagation Neural Network (BPNN) is a feed-forward network consists of input layer, a number of hidden layer and output layer. Scholars have proved that, with a single hidden layer neural network can carry on the arbitrary accuracy to any nonlinear mapping approaching [7]. This paper uses three layer network structure containing a single hidden layer, its topology structure is shown as Figure 1.

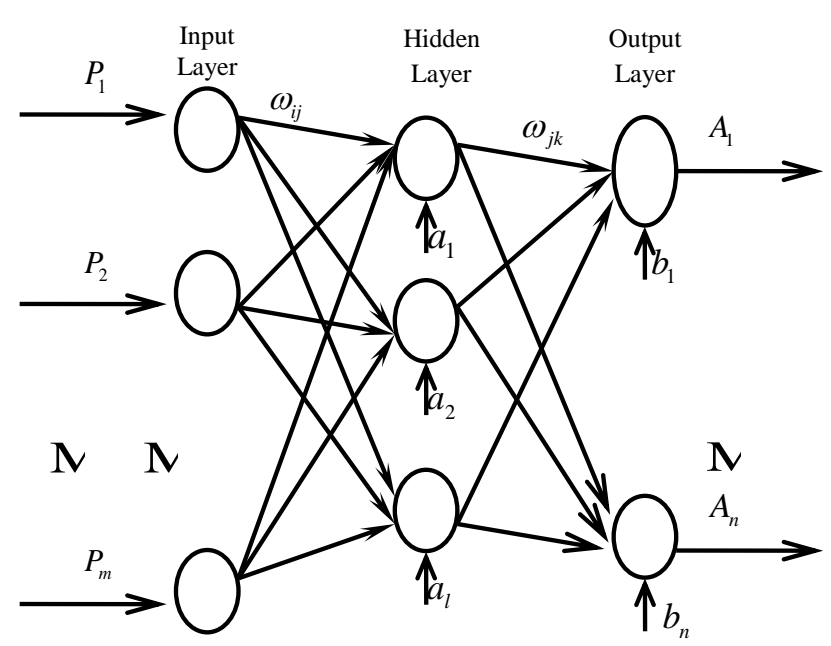

Figure 1. The Topology Structure of Three Layers BPNN

Where $\left(P_{1}, P_{2}, \mathrm{~L}, P_{m}\right)$ is the input vectors of network; $\omega_{i j}$ is the weight vector between input layer and hidden layer; $\left(a_{1}, a_{2}, \mathrm{~L}, a_{l}\right)$ is the threshold vector of hidden layer node; $\omega_{j k}$ is the weight vector between hidden layer and output layer; $A_{1}, A_{2}, \mathrm{~L}, A_{n}$ is the output vectors of network; $\left(b_{1}, \mathrm{~L}, b_{n}\right)$ is the threshold vector of output layer node.

BP network to be trained before application, it has obvious advantages in dealing with non-linear problems. The main features of raining process is information forward propagation and the error back propagation [8]. Forward propagation is the input vector gradually processing through the input layer and hidden layer spread to the output layer. 
Error back propagation means that calculate error value when the forecast output and the expected output is not consistent, and then the error signal transfer through the output layer and hidden layer spread to the input layer to modify the weights and thresholds of each neuron until reach the target. The training flow is shown in Figure 2.

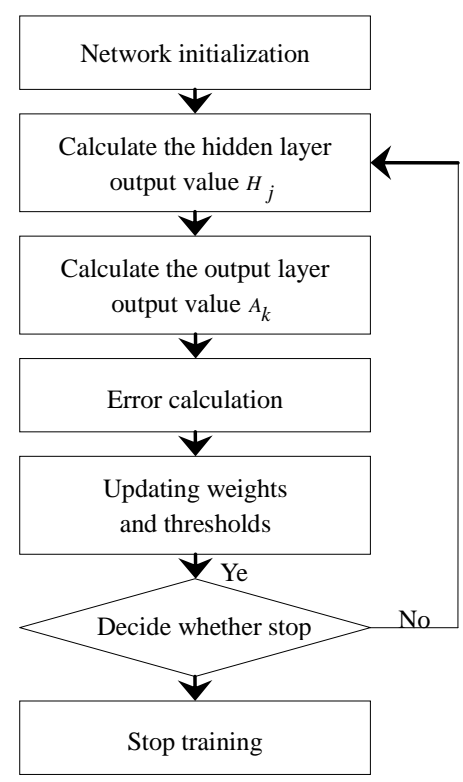

Figure 2. Training Process of BPNN

\section{The Modified of PSO Algorithm}

\subsection{The Basic PSO Algorithm}

PSO algorithm [9] is a kind of optimization algorithm derives from the study of bird feeding behavior, which was first proposed by Dr. Eberhart and Dr. Kennedy in 1995, it is a global optimization technology based on swarm intelligence. The mathematical meaning is: assumed that in M-dimensional search space, there is a population composed with $\mathrm{N}$ particles, set of population is $X=\left(X_{1}, X_{2}, \mathrm{~L}, X_{N}\right)$, where each particle represents a potential optimal solution. The fitness value of each particle position $X_{i}=\left[X_{i 1}, X_{i 2}, \mathrm{~L}, X_{i M}\right]^{T}$ can be calculated by the fitness function, which indicates the pros and cons of the particles. Each particle $V_{i}=\left[V_{i 1}, V_{i 2}, \mathrm{~L}, V_{i M}\right]^{T}$ has a velocity determine its direction and distance of flying. Thus with the position, velocity, and the fitness value can represent characteristics of the particles. First, get a group of particles through random initialization, and then through the iteration to optimization. Each iteration, the particle through individual extremum $P_{i}=\left[P_{i 1}, P_{i 2}, \mathrm{~L}, P_{i M}\right]^{T}$ and global extremum $P_{g}=\left[P_{g 1}, P_{g 2}, \mathrm{~L}, P_{g M}\right]^{T}$ update its velocity and position, update formula can be expressed as follow ${ }^{[10]}$ :

$$
\begin{aligned}
V_{i m}^{k+1} & =\omega V_{i m}^{k}+c_{1} r_{1}\left(P_{i m}^{k}-X_{i m}^{k}\right)+c_{2} r_{2}\left(P_{g m}^{k}-X_{i m}^{k}\right),\left(c_{1} \geq 0, c_{2} \geq 0\right) \\
X_{i m}^{k+1} & =X_{i m}^{k}+V_{i m}^{k+1}
\end{aligned}
$$

Where $V_{i n}$ is the particle's velocity; $X_{i m}$ represents particle's position; $c_{1}$ and $c_{2}$ is the acceleration factor; $r_{1}$ and $r_{2}$ is the random number which distribution between zero and one; $m=1,2, \mathrm{~L}, M, i=1,2, \mathrm{~L}, N$; and $k$ is the number of iterations. 


\subsection{The Modified PSO Algorithm}

In this paper, we adopt adaptive mutation and linear decreasing inertia weight strategies to improve the basic PSO algorithm which can overcome some defects of basic PSO algorithm.

\section{(1) Adaptive mutation}

Learn from the mutation of genetic algorithm, mutation operation is introduced to the basic PSO algorithm. That is, after each of the particle velocity and position update, to re-initialize the particle at a certain probability, based on MATLAB programming code is as follows:

$$
\begin{aligned}
& \text { if rand }>0.9 \\
& \quad k=c e i l(M * \text { rand }) ; \\
& \quad \text { pop }(j, k)=\text { rand; } \\
& \text { end }
\end{aligned}
$$

Where $\mathrm{j}$ is the number of population size; $\mathrm{M}$ is the dimension of particle's velocity vector and position vector.

The diversity of the population can be increased by introducing mutation operation, expand the population search spaces that gradual reduction in iterative process, so as to improve the possibility of finding the optimal value, avoid the particles fall into local optimum.

\section{(2) The setting of linear decreasing inertia weight}

In order to improve the convergence performance of the basic PSO algorithm, linear decreasing inertia weight is added in the velocity update formula, which can be expressed as follows:

$$
\begin{aligned}
& V_{i m}^{k+1}=\omega V_{i m}^{k}+c_{1} r_{1}\left(P_{i m}^{k}-X_{i m}^{k}\right)+c_{2} r_{2}\left(P_{g m}^{k}-X_{i m}^{k}\right), \quad\left(c_{1} \geq 0, c_{2} \geq 0\right) \\
& \omega=\omega_{\text {start }}-\left(\omega_{\text {start }}-\omega_{\text {end }}\right) * k / T_{\text {max }}
\end{aligned}
$$

Where $\omega_{\text {start }}$ is the initial inertia weight; $\omega_{\text {end }}$ is the inertia weight of iteration to the maximum number; $k$ is the number of iterations; and $T_{\max }$ is the maximum iterations. In general, when the inertia weight value $\omega_{\text {sart }}=0.9$ and $\omega_{\text {end }}=0.4$ is the best performance of the algorithm. As the iteration proceeds, the inertia weight linearly decreasing from 0.9 to 0.4 , larger $\omega$ makes the algorithm keeps the good global exploring ability at beginning of iteration, while smaller $\omega$ is conducive to the algorithm maintains a good global exploring ability, thus balance the particles' global search ability and local search ability, improve the convergence performance and accuracy of particle swarm.

The flow chart of modified Particle Swarm Optimization algorithm is shown as Figure 3. 


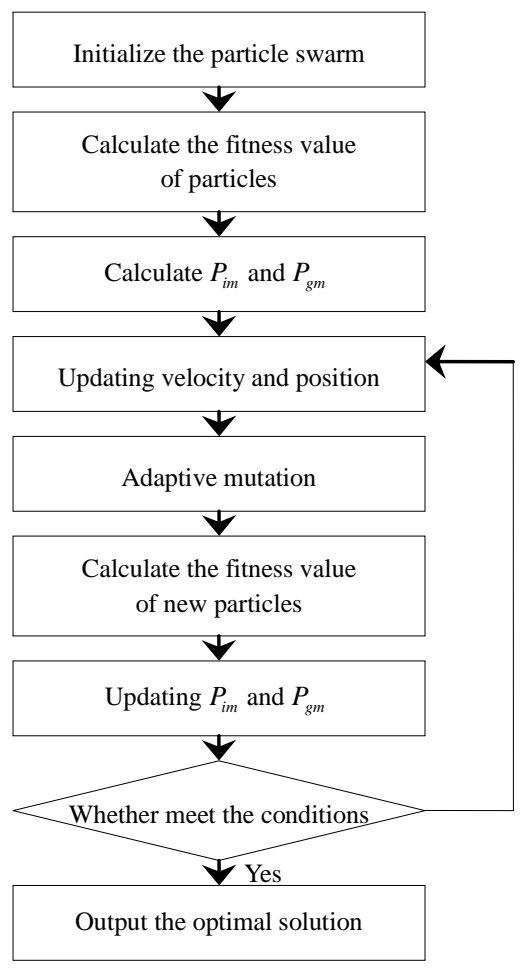

Figure 3. The Flow Chart of Modified Particle Swarm Optimization Algorithm

Initialize the particle swarm is randomly set the initial position $x$ and velocity $V$ of each particle. Fitness value $(F)$ of each particle can be calculated by the fitness function. Determine individual extremum and global extremum according to the initial particle fitness value. Update velocity and position by formula (2), formula (3) and formula (4). Update particle's individual extremum and global extremum according to the fitness value of particle in the new population. If the termination condition (position is good enough or the maximum number of iterations is reached) is reached, output the optimal solution, otherwise return to step 4.

\section{Construction of MPSO-BP Neural Network Assessment Model}

Due to the BP neural network training using the gradient descent method, the learning results depends on the initial weights and thresholds, This can cause oscillation and poor generalization ability of the network, eventually led to the trained network is easy to fall into local optimum and the reliability of the network is poor. MPSO-BP neural network is to use the Modified Particle Swarm Optimization algorithm to find a better network weights and thresholds of network, MPSO algorithm is easy to implement, has a fast convergence speed and better global optimization ability. Therefore, Modified Particle Swarm Optimization algorithm can be used to optimize the BP neural network to improve learning performance and convergence performance of the network, minimize the global error of BP neural network, has a higher prediction precision.

\subsection{Determination Structure of MPSO-BP Neural Network}

We put the earlier analysis of the six main factors which affecting target threat assessment $(V, K, \theta, H, D, C)$ as input samples, Target threat values $(T)$ as the output vector, which can determine the structure of MPSO-BPNN: input layer node 
number $m=6$ and output layer node number is $n=1$, according to the optimal number of hidden layer nodes selection formula ${ }^{[11]} l=\sqrt{(\mathrm{m}+\mathrm{n})}+c$, where $c \in(0,10)$, in this paper, the number of hidden layer nodes is set to 8 , thus building a single hidden layer MPSO-BPNN, which structure is 6-8-1.

\subsection{The Training and Assessment of MPSO-BP Neural Network}

The training and assessment process of MPSO-BPNN are illustrated as follows:

(1) MPSO algorithm parameter setting. According to research in this paper, set the acceleration factor $c_{1}=c_{2}=1.49$; $\omega$ use the linear decreasing inertia weight which shown as Equation (4), where $\omega_{\text {start }}=0.9, \omega_{\text {end }}=0.4$; the number of iterations is 450 ; the size of the population is 65 ; other parameters using the system defaults.

(2) According to the BPNN structure of the previously constructed calculate the length of weights and thresholds, that is $6 \times 8+8 \times 1+8+1=65$. Use PSO algorithm encode the connection weights between layers and the threshold of each node into real vector, represents the particles in population, so the dimension $M$ of the particle's velocity and position vector are 65. Use MATLAB intrinsic function rands $(1,65)$ to generate initial position and velocity of the population.

(3) Compute the fitness value of each particle by the fitness function, get the optimal solution by continuously updating speed and position. According to the actual situation, this paper selected the BP neural network test outputs' mean square error of relative error as fitness function error $=m s e\left(\left(A_{i}-T_{i}\right) . / T_{i}\right)$.

(4) After each of the particle update, to re-initialize the particle at a certain probability, obtained the optimal solution through adaptive mutation and constantly iterative update. Combined with the example, based on MATLAB programming code is as follows:

$$
\begin{aligned}
& \text { if rand }>0.9 \\
& \quad k=\text { ceil }\left(6 *^{*} \text { rand }\right) \text {; } \\
& \quad \text { pop }(j, k)=\text { rand; } \\
& \text { end }
\end{aligned}
$$

(5) The optimal solution of MPSO is assigned to the weights and thresholds of BPNN, and set the operating parameters of network for training. Combined with the actual needs, in this paper, the network training parameters are set as follows: training times is 300 ; the learning rate is 0.1 ; the expected error is $1 \mathrm{e}-005$, other parameters using the system defaults.

(6) Using trained MPSO-BPNN to assessment, and analyze the effect of this model.

The flow chart of MPSO-BPNN algorithm is shown as Figure 4. 


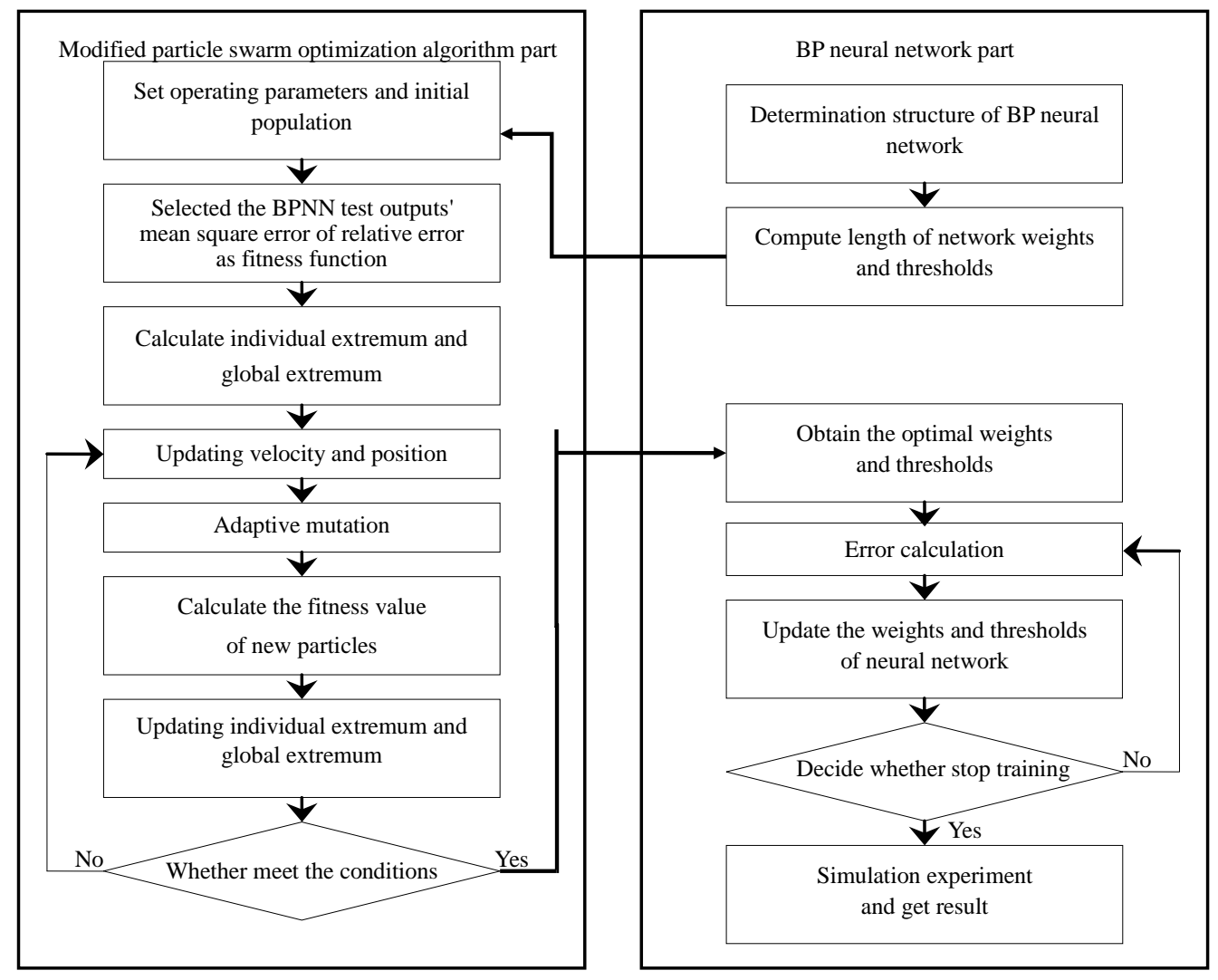

Figure 4. The Flow Chart of MPSO-BPNN Algorithm

\section{Actual Example and Analysis}

We used the six influence factors $(V, K, \theta, H, D, C)$ of target threat values as independent variables and the target threat values $(T)$ as dependent variable. 36 groups of historical statistical data are listed in Table 1, large target, small target and reconnaissance plane each have 12 groups.

Table 1. Sample Data

\begin{tabular}{cccccccc}
\hline nо. & $V(\mathrm{~m} / \mathrm{s})$ & $K$ & $\theta\left({ }^{\circ}\right)$ & $H(\mathrm{~km})$ & $D(\mathrm{~km})$ & $C$ & $T$ \\
\hline 1 & 400 & 8 & 5 & 8 & 140 & 8 & 0.5056 \\
2 & 760 & 8 & 4 & 8 & 120 & 8 & 0.6235 \\
3 & 740 & 8 & 6 & 4 & 110 & 6 & 0.6473 \\
4 & 560 & 8 & 10 & 6 & 150 & 6 & 0.5828 \\
5 & 460 & 8 & 8 & 4 & 200 & 6 & 0.5784 \\
6 & 430 & 8 & 11 & 4 & 130 & 8 & 0.5841 \\
7 & 620 & 8 & 13 & 2 & 320 & 8 & 0.6302 \\
8 & 700 & 8 & 17 & 8 & 310 & 6 & 0.5580 \\
9 & 730 & 8 & 16 & 2 & 260 & 8 & 0.6574 \\
10 & 630 & 5 & 5 & 8 & 160 & 6 & 0.5943 \\
11 & 720 & 5 & 7 & 4 & 300 & 8 & 0.7336 \\
12 & 660 & 5 & 17 & 6 & 270 & 8 & 0.6424 \\
13 & 970 & 5 & 3 & 6 & 310 & 8 & 0.8253 \\
14 & 830 & 5 & 12 & 8 & 160 & 8 & 0.7285 \\
15 & 900 & 5 & 17 & 8 & 310 & 8 & 0.7052 \\
16 & 1010 & 5 & 5 & 8 & 190 & 8 & 0.8078 \\
17 & 1080 & 5 & 8 & 6 & 270 & 8 & 0.8306 \\
18 & 1250 & 5 & 13 & 4 & 190 & 6 & 0.8789 \\
19 & 90 & 3 & 2 & 8 & 220 & 4 & 0.3581 \\
20 & 95 & 3 & 9 & 4 & 190 & 4 & 0.3781 \\
21 & 90 & 3 & 17 & 4 & 180 & 4 & 0.3504
\end{tabular}




$\begin{array}{lccccccc}22 & 96 & 3 & 10 & 6 & 310 & 4 & 0.3427 \\ 23 & 100 & 3 & 13 & 4 & 220 & 4 & 0.3624 \\ 24 & 105 & 3 & 4 & 8 & 160 & 4 & 0.3706 \\ 25 & 109 & 3 & 13 & 4 & 270 & 4 & 0.3623 \\ 26 & 105 & 3 & 17 & 2 & 140 & 2 & 0.3605 \\ 27 & 120 & 3 & 11 & 2 & 210 & 2 & 0.3966 \\ 28 & 540 & 8 & 3 & 6 & 340 & 8 & 0.6104 \\ 29 & 440 & 8 & 7 & 4 & 320 & 6 & 0.5823 \\ 30 & 460 & 8 & 15 & 4 & 260 & 6 & 0.5563 \\ 31 & 1100 & 5 & 15 & 4 & 320 & 8 & 0.8424 \\ 32 & 560 & 5 & 5 & 6 & 230 & 8 & 0.6026 \\ 33 & 710 & 5 & 11 & 4 & 320 & 8 & 0.7206 \\ 34 & 115 & 3 & 14 & 4 & 330 & 4 & 0.3587 \\ 35 & 90 & 3 & 13 & 2 & 320 & 4 & 0.3897 \\ 36 & 100 & 3 & 8 & 6 & 250 & 2 & 0.3462\end{array}$

This paper selects 1 to 27 groups as neural network training data and 28 to 36 groups as test data. To illustrate the effectiveness of the proposed model, respectively using BPNN assessment model and PSO-BPNN assessment model compared with MPSO-BPNN assessment model, simulation results are shown in Figure 5. Comparison of MPSO algorithm and basic PSO algorithm, we can see the optimal individual fitness value changes in the process of optimization, which has shown in Figure 6.

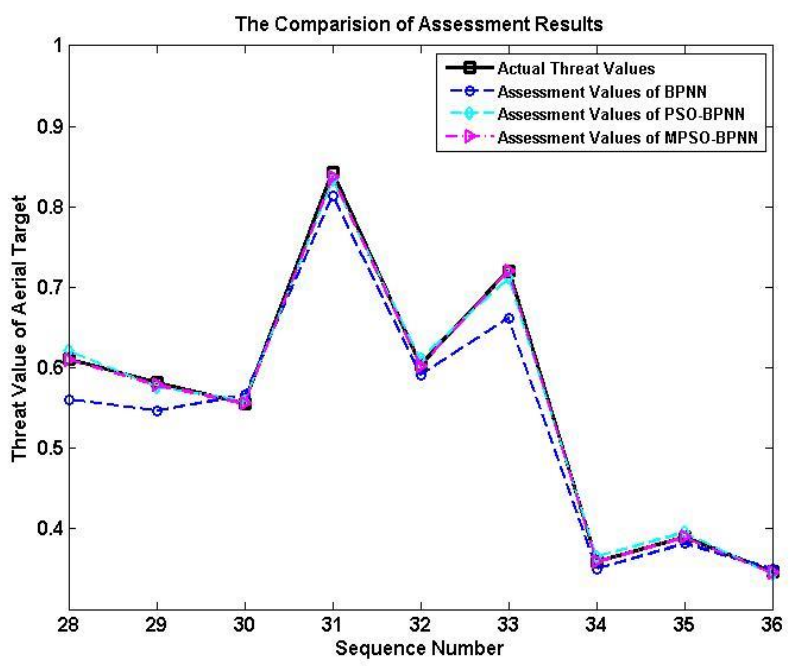

Figure 5. Comparison of Assessment Results

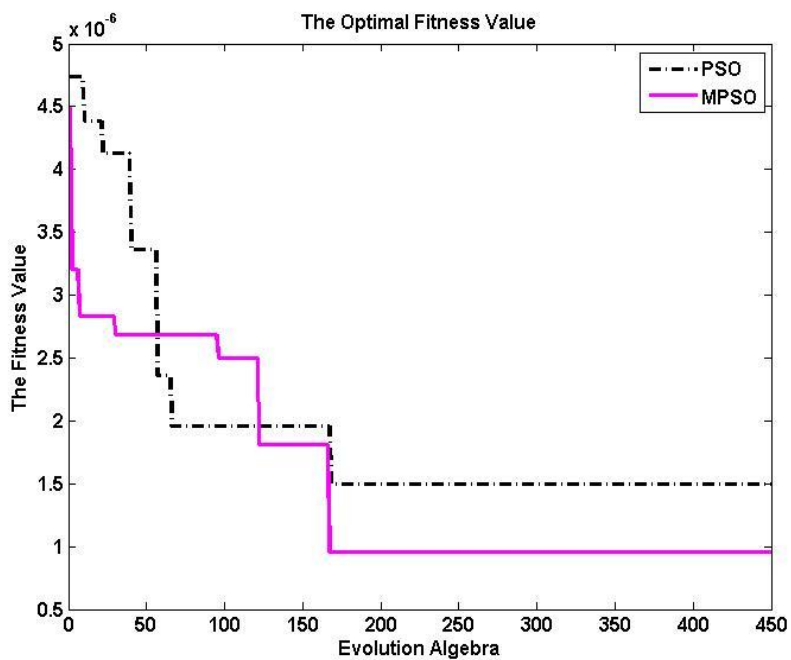

Figure 6. Curve of Best Individual Fitness Value Changes

It can be seen from Figure 6, The MPSO algorithm's fitness value after 167 iterations 
has been better than the basic PSO algorithm's optimal fitness value after 450 iterations, shows that the convergence speed of the MPSO algorithm is faster and the convergence accuracy is higher.

By comparative analysis the actual threat value and the assessment value of the above-mentioned three kinds of neural network model, get the error curve and the relative error curve, which has shown in Figure 7 and Figure 8, specific data are shown in Table 2.

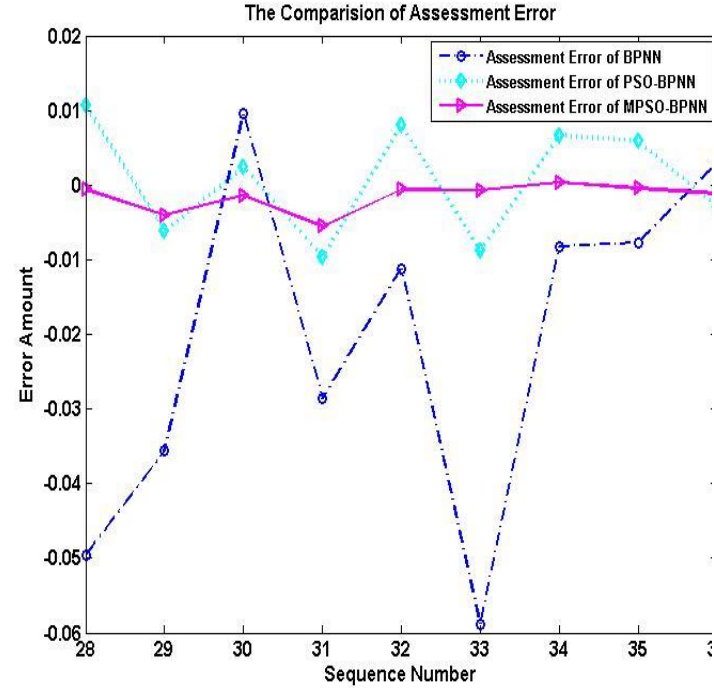

Figure 7. Assessment Error Curve

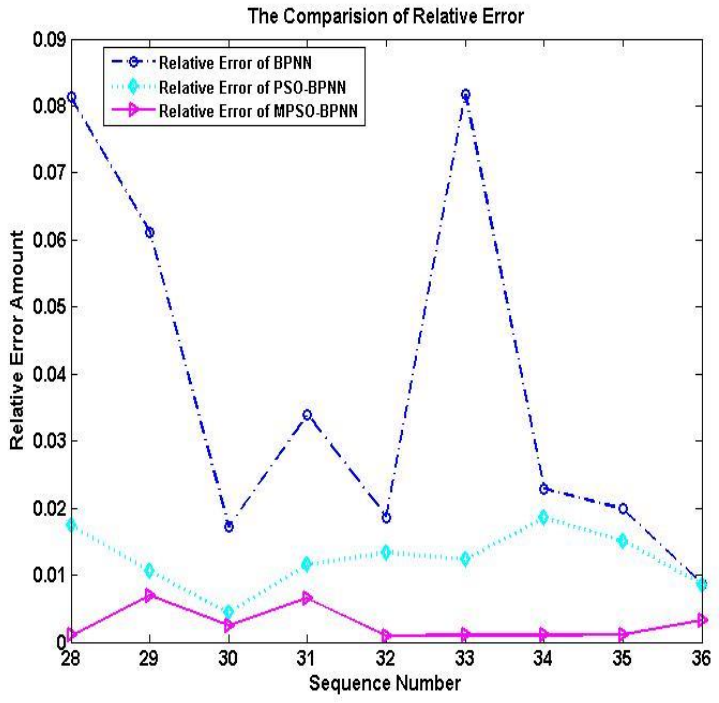

Figure 8. Relative Error Curve

Table 2. Error Analysis

\begin{tabular}{|c|c|c|c|c|c|c|c|c|c|c|}
\hline \multicolumn{2}{|c|}{ Sequence number } & 28 & 29 & 30 & 31 & 32 & 33 & 34 & 35 & 36 \\
\hline \multicolumn{2}{|c|}{ actual threat value } & 0.6104 & 0.5823 & 0.5563 & 0.8424 & 0.6026 & 0.7206 & 0.3587 & 0.3897 & 0.3462 \\
\hline \multirow{3}{*}{ BPNN } & assessment value & 0.5608 & 0.5467 & 0.5658 & 0.8138 & 0.5914 & 0.6617 & 0.3505 & 0.3820 & 0.3492 \\
\cline { 2 - 10 } & error amount & -0.0496 & -0.0356 & 0.0095 & -0.0286 & -0.0112 & -0.0589 & -0.0082 & -0.0077 & 0.0030 \\
\cline { 2 - 11 } & relative error amount & 0.0813 & 0.0611 & 0.0171 & 0.0340 & 0.0186 & 0.0817 & 0.0229 & 0.0198 & 0.0087 \\
\hline \multirow{3}{*}{ PSO-BPNN } & assessment value & 0.6210 & 0.5762 & 0.5587 & 0.8327 & 0.6107 & 0.7118 & 0.3653 & 0.3956 & 0.3433 \\
\cline { 2 - 10 } & error amount & 0.0106 & -0.0061 & 0.0024 & -0.0097 & 0.0081 & -0.0088 & 0.0066 & 0.0059 & -0.0029 \\
\cline { 2 - 11 } & relative error amount & 0.0174 & 0.0105 & 0.0043 & 0.0115 & 0.0134 & 0.0122 & 0.0184 & 0.0151 & 0.0084 \\
\hline \multirow{3}{*}{ MPSO-BPNN } & assessment value & 0.6098 & 0.5783 & 0.5549 & 0.8369 & 0.6020 & 0.7199 & 0.3591 & 0.3892 & 0.3451 \\
\cline { 2 - 10 } & error amount & -0.0006 & -0.0040 & -0.0014 & -0.0055 & -0.0006 & -0.0007 & 0.0004 & -0.0005 & -0.0011 \\
\cline { 2 - 10 } & relative error amount & 0.0010 & 0.0069 & 0.0025 & 0.0065 & 0.0010 & 0.0010 & 0.0011 & 0.0012 & 0.0032 \\
\hline
\end{tabular}

According to the Table 2, the sum of the relative error amount of BPNN is 0.3452 , the sum of the relative error amount of PSO-BPNN is 0.1112 and the sum of the relative error amount of MPSO-BPNN is 0.0244. Simulation results show that the efficiency of the MPSO algorithm is improved and has better performance in reaching the global optimum, the assessment accuracy of MPSO-BP neural network is higher than the BP neural network and the PSO-BP neural network, a more accurate assessment results are obtained, can be used as a new method to assess threat of aerial target.

\section{Conclusion}

This paper studies the problem of aerial target threat assessment based on Modified PSO optimized BP Neural Network and construct the MPSO-BP neural network assessment model, which can be effectively overcomes some defects of BP neural network and PSO algorithm. Through the analysis of the numerical example proves that this method has better ability to assess, it can quickly and accurately assess the air target threat values, provide support for task allocation and tactical decisions. 


\section{References}

[1] M. Cheng, D. Zhou and K. Zhang, "Threat Assessment of Target Based on a Hybrid Multi-Attribute Decision-Making Method", Electronic Optics \& Control, vol. 1, no. 17, (2010).

[2] S. Yan, H. Liu and T. Tu, "A Threat Sequencing Method for Aerial Infrared Multi-Target", Fire Control \& Command Control, vol. 6, no. 39, (2014).

[3] L. Xiao, J. Huang and Z. Xu, "Cooperative attack decision for BVR air combat based on neural network", Journal of Shenyang University of Technology, vol. 3, no. 35, (2013).

[4] S. Nian, Y. Lin and R. Chen, "Research on Aerial Threat Evaluation Model Simulation for Formation Based on Improved BP Algorithm", Computer \& Digital Engineering, vol. 12, no. 39, (2011).

[5] H. Chen, K. Zhang and J. Cao, "Research on Aerial Threat Evaluation Model Simulation for Formation Based on Improved BP Algorithm", Application Research of Computers, vol. 3, no. 29, (2012).

[6] S. Wei and X. Fei, "Multiple Attribute Decision Theoretic Method and Its Application in $\mathrm{C}^{3} \mathrm{I}$ System", National Defend Industry Press, Peking, vol. 3, (1998), pp.86-91.

[7] W. Wang, J. Li and F. Zhang, "Simulation of solar radiation in Lanzhou based on BP neural network", Journal of Arid Land Resources and Environment, vol. 2, no. 28, (2014).

[8] C. Yuping, Z. Yuan and B. Zhou, "Application of back propagation neural networks with optimization of genetic algorithms to landslide hazard prediction”, Hydrogeology \& Engineering Geology, vol. 1, no. 39, (2012).

[9] J. Kennedy and R. C. Eberhart, "Particle swarm optimization", Proceedings of IEEE International Conference on Neural Networks, November; Perth, Australia, (1995).

[10] Q. Feng, "Particle Swarm Optimization and its industrial applications", Science Press, Peking, (2013), vol. 2, pp.28-31.

[11] G. Fei, "Super Learning Manual Based on MATLAB Intelligent Algorithm", Posts \& Telecom Press, Peking, vol. 3, (2014), pp.40-43.
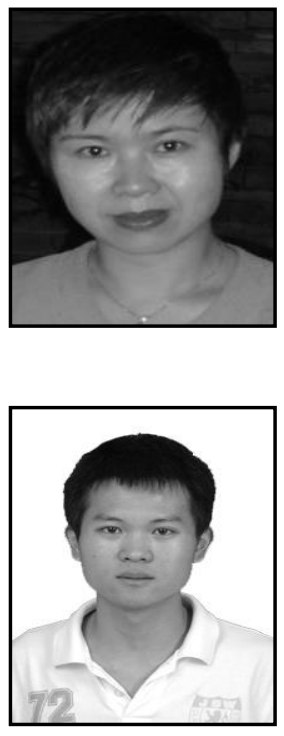

\section{Authors}

Xia Chen, she was born in 1962, she is a professor in Shenyang Aerospace University. The main research direction: Air Materiel Management, flight control, etc.

E-mail:xiachen1108@163.com
Tuo Wang, he was born in 1989 , he is a graduate student in Shenyang Aerospace University. The main research direction: Air Materiel Management, intelligent decision, etc.

E-mail:wangtuo316@163.com 
International Journal of Control and Automation

Vol. 10, No. 2 (2017) 